\title{
LONG-TERM DECREASE AND CYCLIC VARIATION IN THE ORBITAL PERIOD OF THE ECLIPSING DWARF NOVA V2051 OPH
}

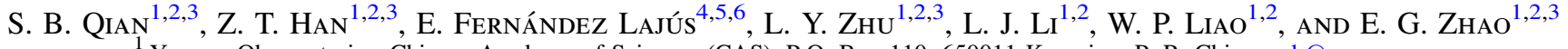 \\ Yunnan Observatories, Chinese Academy of Sciences (CAS), P.O. Box 110, 650011 Kunming, P. R. China; qsb@ynao.ac.cn \\ ${ }^{2}$ Key laboratory of the structure and evolution of celestial objects, Chinese Academy of Sciences, P.O. Box 110, 650011 Kunming, P. R. China \\ ${ }^{3}$ University of the Chinese Academy of Sciences, Yuquan Road 19\#, Sijingshang Block, 100049 Beijing, P. R. China \\ ${ }^{4}$ Facultad de Ciencias Astronómicas y Geofísicas, Universidad Nacional de La Plata, Paseo del Bosque s/n, 1900, La Plata, Pcia. Bs. As., Argentina \\ ${ }^{5}$ Instituto de Astrofísica de La Plata (CCT La plata-CONICET/UNLP), Argentina \\ Received 2015 July 22; accepted 2015 October 12; published 2015 November 5
}

\begin{abstract}
V2051 Oph is a deeply eclipsing dwarf nova with an orbital period below the period gap of cataclysmic variables (CVs). It has been photometrically monitored since 2008 June and 24 mid-eclipse times of the white dwarf have been obtained. The changes in the orbital period are investigated using all of the available mid-eclipse times. A

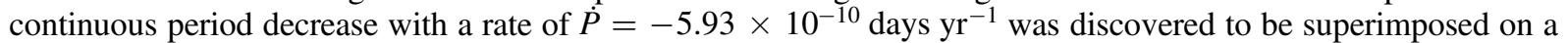
periodic variation with a small amplitude of 0.000329 and a period of 21.64 years. The standard theory predicted that the evolution of CVs below the period gap is driven by gravitational radiation. However, angular momentum loss (AML) via gravitational radiation is insufficient to explain this decrease, and additional AML via magnetic braking that is about five times the gravitational radiation rate is required. This is consistent with the theoretical requirement indicating that magnetic braking of the fully convective star is not completely stopped. The cyclic oscillation was interpreted as the variation of the arriving eclipse time via the presence of a third body because the required energy for the Applegate mechanism is much larger than that radiated from the secondary in 10 years. Its mass is derived as $M_{3} \sin i^{\prime}=7.3( \pm 0.7)$ Jupiter mass. For orbital inclinations $i^{\prime} \geqslant 30^{\circ} .3$, it would be a planetary object. The giant circumbinary planet is orbiting around V2051 Oph at an orbital separation of about 9.0 astronomical units (AU) in an eccentric orbit $\left(e^{\prime}=0.37\right)$. These conclusions support the ideas that some planets could survive stellar late evolution and that dwarf novae are also planetary hosting stars.
\end{abstract}

Key words: binaries: eclipsing - planetary system - stars: dwarf nova - stars: evolution - stars: individuals (V2051 Oph)

\section{INTRODUCTION}

The final fate of extrasolar planets orbiting main-sequence (MS) stars is of interest as it is well know that planets are common in the Solar neighborhood (Howard et al. 2010). Several theoretical investigations showed that some of these planets can survive the post-MS evolution of the hosting stars (e.g., Villaver \& Livio 2007; Carlberg et al. 2009; Nordhaus et al. 2010; Mustill \& Villaver 2012). Finding planetary companions to white dwarfs (WDs) can provide insight into the fate of planetary systems around Sun-like stars and can shed light on the interplay between substellar objects and late stellar evolution (e.g., the red giant and asymptotic giant stages). Several imaging surveys were proposed to search for planets orbiting WDs with limits typically from 5 to $10 M_{\text {Jup }}$ (e.g., Mullally et al. 2007; Farihi et al. 2008; Burleigh et al. 2008; Hogan et al. 2009). However, to date, there have been no reported planet detections around WDs. The reason for the lack of detections may be a selection effect of the targets (Kilic et al. 2007). When a white dwarf is a component of an eclipsing binary, the eclipse times can be determined with high precision because of the small size and compact structure $\left(R \sim 0.01 R_{\odot}\right)$ of the white dwarf. Therefore, its small wobbles caused by the presence of other substellar companions can be discovered by measuring the eclipse times. As the eclipsing binary orbits the barycenter of the triple system, the arrival eclipse time varies

\footnotetext{
${ }^{6}$ Visiting Astronomer, Complejo Astronómico El Leoncito operated under agreement between the Consejo Nacional de Investigaciones Científicas y Técnicas de la República Argentina and the National Universities of La Plata, Córdoba and San Juan.
}

cyclically. This method has successfully been used to detect extrasolar planets around subdwarf B-type stars and white dwarfs. Several planets were reported to be orbiting eclipsing white dwarf binaries such as QS Vir (Qian et al. 2010b; Almeida \& Jablonski 2011), NN Ser (Marsh et al. 2014), RR Cae (Qian et al. 2012a), DP Leo (Qian et al. 2010a; Beuermann et al. 2011), and HU Aqr (Qian et al. 2011; Goździewski et al. 2015).

Several observational campaigns were planned to attempt to detect extrasolar planets orbiting close binary systems, including white dwarf binaries, using the timings of the light minima (e.g., Backhaus et al. 2012; Pribulla et al. 2012). To search for planets and brown dwarfs around evolved binary stars, since 2006 we have monitored some evolved binary systems. We are interested in several kinds of binary stars: (1) detached WD $+\mathrm{dM}$ binaries such as NN Ser, QS Vir, and RR Cae (Qian et al. 2009a, 2010b, 2012a); (2) subdwarf B-type (sdB) eclipsing binaries such as HS0705+6700 and NY Vir (e.g., Qian et al. 2009c, 2012b, 2013); (3) magnetic CVs such as DP Leo (Qian et al. 2010a); and (4) deeply eclipsing cataclysmic variables (CVs) including some nova-like CVs and dwarf novae (e.g., Qian et al. 2009b). Here, we report the period variations of the eclipsing dwarf nova V2051 Oph obtained through photometric monitoring since 2008.

As mass transfer binaries, CVs must continuously undergo angular momentum loss (AML) to maintain the mass transfer process (e.g., Warner 1995). In this way, the mass of the secondary is continuously decreasing and the orbit of the system is shrinking simultaneously (Andronov et al. 2003). According to the standard theory of CVs, the mechanism 
driving AML is magnetic braking (MB) for systems with $P>3$ hours, and the period gap of CVs (the apparent scarcity of CVs between 3 and $2 \mathrm{hr}$ ) was interpreted as the end of MB when the secondary becomes fully convective. As for systems below the period gap, AML is driven by gravitational radiation (GR; Rappaport et al. 1983; Spruit \& Ritter 1983). However, the disrupted MB causes the theory to encounter the "period minimum" problem, i.e., the sharp cut-off of the period distribution near 78 minutes cannot be explained (Kolb 1993; King et al. 2002). Observationally, in addition to searching for planets, those long-term photometric data could in principle be used to investigate the secular evolution of CVs by analyzing changes in the orbital periods of eclipsing CVs.

As one of the subtypes of CVs, dwarf novae are close binary systems where mass transfer take place from a low-mass lobefilling secondary to a white dwarf via accretion, but with a very low rate of mass transfer (Warner 1995). This subclass of CVs is characterized by recurrent and large-amplitude outbursts with $\Delta m \sim 3-5 \mathrm{mag}$ and a typical duration of 5-10 days, which is explained by the structure change of their disks (Saito \& Baptista 2006). With an orbital period of about 90 minutes (below the CV period gap) and recurrent outbursts, V2051 Oph is one of a few ultrashort-period dwarf novae (Baptista et al. 1998), but it is distinguished from the other dwarf novae by showing flickering activity and sparse outbursts (Baptista et al. 2007). The mass and radius of the secondary in V2051 Oph are $M_{2}=0.15 M_{\odot}$ and $R_{2}=0.15 R_{\odot}$, respectively. This should be a fully convective M-type star and it seems to obey a zero-age main-sequence (ZAMS) mass-radius relationship (Baptista et al. 1998). This dwarf nova was discovered in 1972 and shows very deep eclipses $(\Delta B \sim 2.5$ magnitudes; Sanduleak 1972; Baptista et al. 2007). This means that eclipses provide a benchmark in time and could be used to measure the orbital period and its rate of change with high precision by analyzing the observed - calculated $(O-C)$ diagram. Therefore, it is one of the most promising systems for checking the evolutionary theory of CVs through the determination of period changes. In the paper, the changes in the orbital period of V2051 Oph are analyzed. A continuous decrease was found to be superimposed on a cyclic change. Then both the evolutionary states of CVs and the presence of a giant planet are discussed.

\section{CCD PHOTOMETRIC MONITORING AND NEW MID-ECLIPSE TIMES}

Photometric monitoring of V2051 Oph began on 2008, June 30 using the 1.0-m reflecting telescope at the Yunnan Observatories. A PI1024 TKB CCD camera mounted to the telescope was used. The size of each pixel was 0.38 arcsec and the effective field of view was about $6.5 \times 6.5$ square arcmin at the Cassegrain focus. During the observations, the R-band filter was used. This binary was later monitored with the 2.4-m telescope at the Lijiang observational station of Yunnan observatories and the 0.6-m Helen Sawyer Hogg (HSH) telescope at CASLEO, Argentina. Starting on 2011, April 3, V2051 Oph was continuously monitored with the 2.15-m Jorge Sahade telescope (JST) at Complejo Astronómico El Leoncito (CASLEO), San Juan, Argentina. A Roper Scientific, Versarray 1300 B camera with a thinned EEV CCD36-40 de $1340 \times 1300$ pix CCD chip was used. To obtain high-precision data and to improve the time resolution of the observations, no filters were applied during the observations. All of the CCD images were

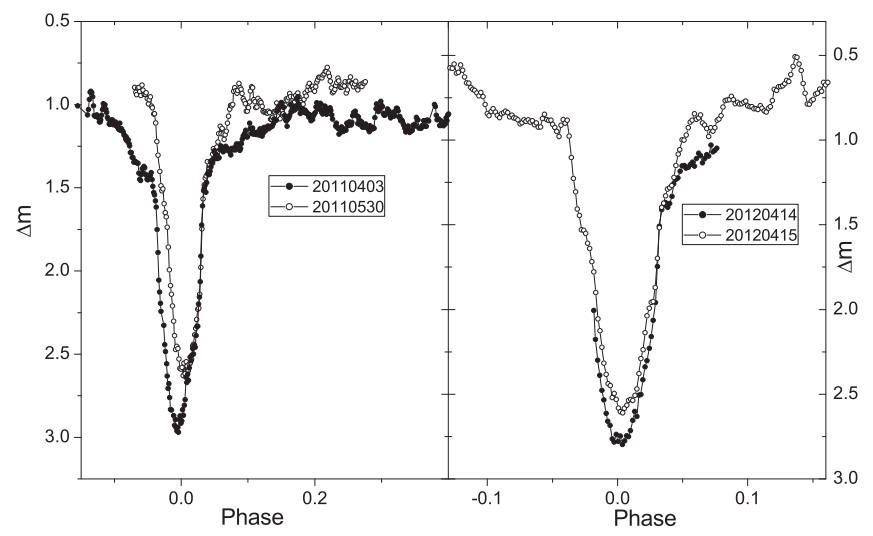

Figure 1. Comparison of eclipse profiles observed in 2011 and 2012 using the 2.15-m Jorge Sahade telescope.

analyzed using PHOT (measures magnitudes for a list of stars) from the aperture photometry package of IRAF. ${ }^{7}$

Two eclipse profiles observed in 2011 with JST are shown in the left panel of Figure 1. The phases of those observations were computed using the linear ephemeris,

$$
\text { Min. I = BJD 2456858.55900 + 0.0624278634 ×E, }
$$

where BJD 2456858.55900 is the initial epoch and one of the mid-eclipse times (listed in Table 1). The orbital period, 0.0624278634 , is from Baptista et al. (2003). For comparison, two eclipse profiles obtained in 2012 are plotted in the right panel. As shown in the two panels, the eclipse profile is variable with time even from night to night. Some intrinsic and random brightness fluctuations of $0.01-0.25$ mag on timescales from seconds to a few minutes, i.e., brightness flickering, are seen. The eclipse profiles observed in 2014 are plotted in Figure 2. As displayed in the figure, in addition to eclipse profile changes and flickering, the light curves show brightness state variations. This brightness changes are caused by variations of mass transfer rate from the secondary to the primary component.

The brightness of the binary system dropped by about one magnitude from July 19 to 20 indicating that a dwarf nova outburst took place in 2014 July. This can be seen more clearly in Figure 3 where mean out-of-eclipse brightness is shown by averaging the observations outside the eclipse. The quick decrease of the brightness indicates that during this time interval the dwarf nova may be in the decline stage of the outburst. Those open pentagrams in Figure 2 refer to the light curve observed during the outburst (on July 19). It is found that the high-frequency flickering disappeared in the light curve. This supports the idea that the high-frequency flickering is produced in the accretion disk showing a radial distribution.

As shown in Figures 1 and 2, the eclipse profile is variable, indicating that the hot spot and accretion disk vary with time. The ingress and egress of the hot spot depend on the accretion disk radius, and thus those times are variable with time. On the other hand, the ingress and egress times of the white dwarf are stable features. Therefore, we determined mid-eclipse times using the mid-ingress and mid-egress times of the white dwarf eclipse with the derivative technique (Wood et al. 1985). In this

\footnotetext{
IRAF (Image Reduction and Analysis Facility) is a collection of software written at the National Optical Astronomy Observatory (NOAO) geared toward the reduction of astronomical images in pixel array form.
} 
Table 1

New CCD Mid-eclipse Times of V2051 Oph

\begin{tabular}{|c|c|c|c|c|c|c|c|}
\hline Min.(HJD) & Min.(BJD) & $E$ & $O-C$ & Duration & Errors & Filters & Telescopes \\
\hline 2454648.11233 & 2454648.11306 & 182645 & -0.00099 & 0.00449 & 0.00015 & $\mathrm{R}$ & $1 \mathrm{~m}$ \\
\hline 2454656.10287 & 2454656.10360 & 182773 & -0.00104 & 0.00451 & 0.00015 & $\mathrm{R}$ & $1 \mathrm{~m}$ \\
\hline 2454678.07767 & 2454678.07840 & 183125 & -0.00103 & 0.00445 & 0.00015 & $\mathrm{R}$ & $1 \mathrm{~m}$ \\
\hline 2454681.69935 & 2454681.69932 & 183183 & -0.00092 & 0.00463 & 0.00020 & $\mathrm{~N}$ & $60 \mathrm{c} \mathrm{m}$ \\
\hline 2454892.45570 & 2454892.45568 & 186559 & -0.00103 & 0.00440 & 0.00006 & $\mathrm{R}$ & $2.4 \mathrm{~m}$ \\
\hline 2454893.39205 & 2454893.39203 & 186574 & -0.00110 & 0.00441 & 0.00004 & $\mathrm{R}$ & $2.4 \mathrm{~m}$ \\
\hline 2455654.82474 & 2455654.82474 & 198771 & -0.00104 & 0.00442 & 0.00006 & $\mathrm{~N}$ & $2.15 \mathrm{~m}$ \\
\hline 2455709.76127 & 2455709.76127 & 199651 & -0.00102 & 0.00445 & 0.00005 & $\mathrm{~N}$ & $2.15 \mathrm{~m}$ \\
\hline 2455710.76006 & 2455710.76006 & 199667 & -0.00108 & 0.00443 & 0.00005 & $\mathrm{~N}$ & $2.15 \mathrm{~m}$ \\
\hline 2455711.75887 & 2455711.75887 & 199683 & -0.00112 & 0.00443 & 0.00005 & $\mathrm{~N}$ & $2.15 \mathrm{~m}$ \\
\hline 2456031.70179 & 2456031.70180 & 204808 & -0.00099 & 0.00444 & 0.00005 & $\mathrm{~N}$ & $2.15 \mathrm{~m}$ \\
\hline 2456032.70064 & 2456032.70065 & 204824 & -0.00099 & 0.00443 & 0.00005 & $\mathrm{~N}$ & $2.15 \mathrm{~m}$ \\
\hline 2456746.87548 & 2456746.87548 & 216264 & -0.00091 & 0.00444 & 0.00005 & $\mathrm{~N}$ & $2.15 \mathrm{~m}$ \\
\hline 2456747.74943 & 2456747.74943 & 216278 & -0.00095 & 0.00443 & 0.00005 & $\mathrm{~N}$ & $2.15 \mathrm{~m}$ \\
\hline 2456857.68503 & 2456857.68503 & 218039 & -0.00082 & 0.00441 & 0.00005 & $\mathrm{~N}$ & $2.15 \mathrm{~m}$ \\
\hline 2456858.55900 & 2456858.55900 & 218053 & -0.00084 & 0.00444 & 0.00005 & $\mathrm{~N}$ & $2.15 \mathrm{~m}$ \\
\hline 2456859.55770 & 2456859.55770 & 218069 & -0.00098 & 0.00444 & 0.00005 & $\mathrm{~N}$ & $2.15 \mathrm{~m}$ \\
\hline 2456873.54155 & 2456873.54155 & 218293 & -0.00097 & 0.00443 & 0.00005 & $\mathrm{~N}$ & $2.15 \mathrm{~m}$ \\
\hline 2456875.60165 & 2456875.60166 & 218326 & -0.00099 & 0.00448 & 0.00005 & $\mathrm{~N}$ & $2.15 \mathrm{~m}$ \\
\hline 2456892.58206 & 2456892.58207 & 218598 & -0.00096 & 0.00442 & 0.00005 & $\mathrm{~N}$ & $2.15 \mathrm{~m}$ \\
\hline 2456944.52198 & 2456944.52198 & 219430 & -0.00103 & 0.00449 & 0.00008 & $\mathrm{~N}$ & $2.15 \mathrm{~m}$ \\
\hline 2457145.29001 & 2457145.29001 & 222646 & -0.00101 & 0.00446 & 0.00015 & $\mathrm{~N}$ & $1 \mathrm{~m}$ \\
\hline 2457190.73764 & 2457190.73763 & 223374 & -0.00087 & 0.00443 & 0.00005 & $\mathrm{~N}$ & $2.15 \mathrm{~m}$ \\
\hline 2457191.73646 & 2457191.73645 & 223390 & -0.00089 & 0.00443 & 0.00005 & $\mathrm{~N}$ & $2.15 \mathrm{~m}$ \\
\hline
\end{tabular}

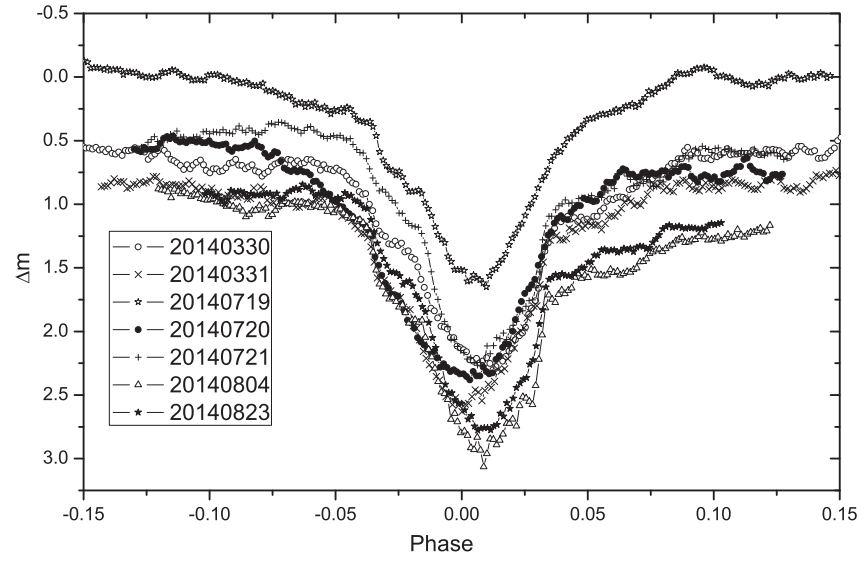

Figure 2. Eclipse profiles observed in 2014. Both the eclipse profile and the out-of-eclipse brightness are variable with time.

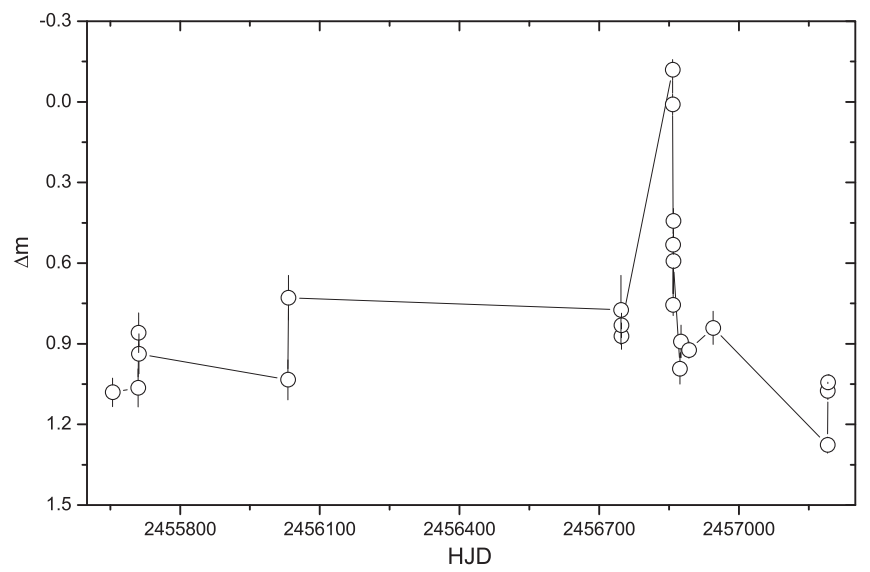

Figure 3. Variation of the out-of-eclipse brightness from 2011 to 2015. Apart from normal brightness state changes, an outburst occurred in 2014 July. The photometry was performed without filters.

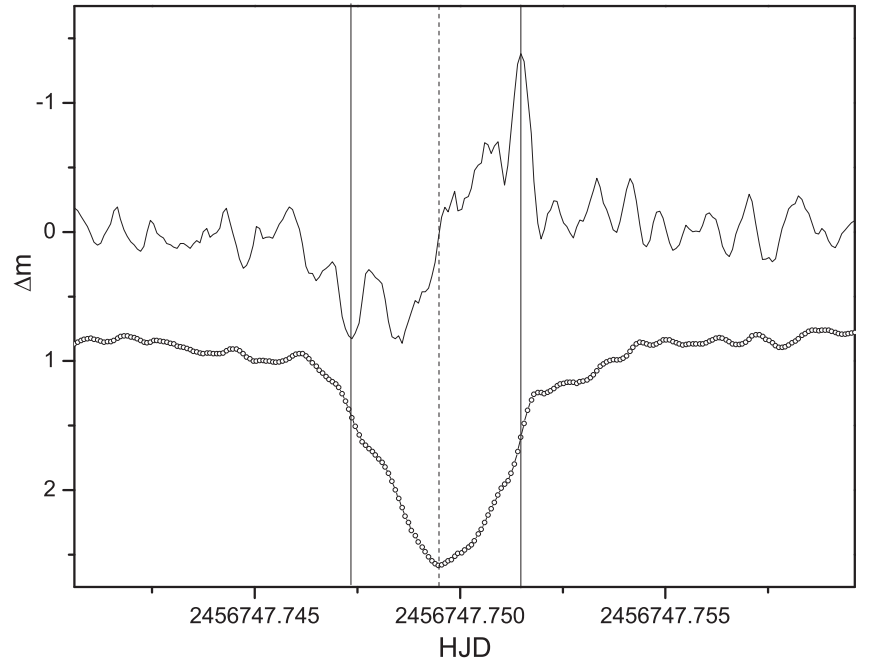

Figure 4. Example of determining mid-eclipse times. The two solid lines refer to the mid-ingress and mid-egress times of the white dwarf component, while the dashed line refers to the mid-eclipse time (see text for details).

method, each light curve was smoothed with a median filter and its numerical derivative was computed. The derivative curve was then analyzed using an algorithm which identifies the extreme points, i.e., the mid-ingress/egress times of the white dwarf. Finally, the mid-eclipse times were derived by averaging the mid-ingress and mid-egress times (Wood et al. 1985). One procedure for measuring the times of the WD ingress and egress is displayed in Figure 4. The determined mid-eclipse times are listed in Table 1 including those in HJD and BJD. To check whether or not the mideclipse times of the WD were determined correctly, the durations of the eclipses were derived and are listed in the fifth column of Table 1. As shown in the table, the durations are constant within the error, indicating that the method is reliable 


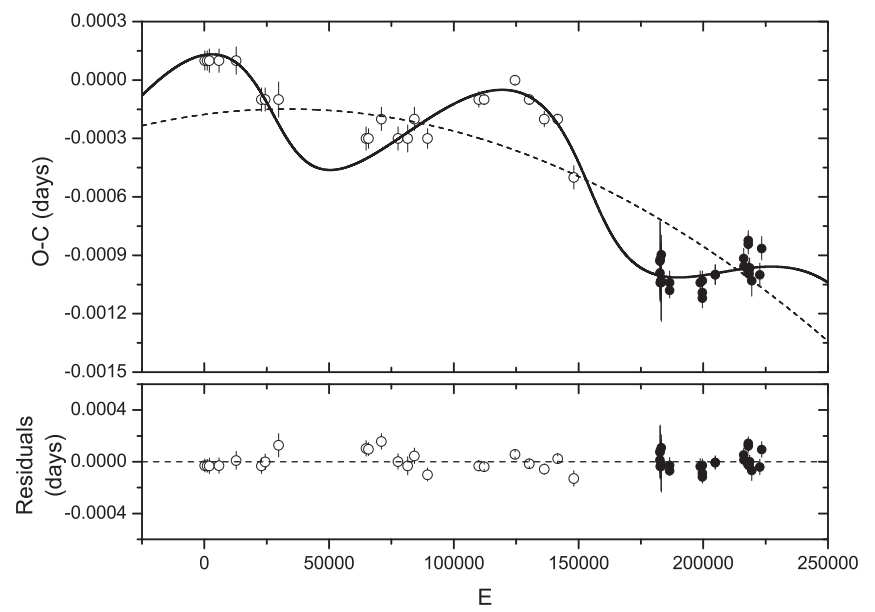

Figure 5. $O-C$ diagram of V2051 Oph with respect to the linear ephemeris of Equation (1). To form the diagram, 24 newly determined mid-eclipse times were used. Open circles refer to the data compiled from the literature, while filled circles refer to our new eclipse times. We show that a simple cyclic change alone cannot fit all of the observations well, indicating the presence of a long-term period decrease (the dashed line). The solid line refers to the combination of the continuous decrease and the cyclic variation. After subtraction of both the continuous decrease and the cyclic variation, the residuals are shown in the lower panel.

for computing those mid-eclipse times. The filters used during the observations are shown in the seventh column where " $N$ " indicates that no filters were used. " $1 \mathrm{~m}$ " and " 2.4 " in the seventh column of the table refer to the $1.0-\mathrm{m}$ and the $2.4-\mathrm{m}$ telescopes in Yunnan observatories, while " $60 \mathrm{~cm}$ " and "2.15 m" refer to the 0.6-m Helen Sawyer Hogg telescope and the 2.15-m Jorge Sahade telescope in Argentina, respectively.

\section{ANALYSIS OF THE $O-C$ DIAGRAM OF V2051 OPH}

Since its discovery, V2051 Oph has been continuously photometrically monitored. Plenty of homogeneous eclipse times have been accurately determined, and a small-amplitude cyclic variation in the orbital period has been reported with a period of 22 years (Baptista et al. 2003). To confirm the cyclic change and to search for long-term period change, the binary system has been monitored since 2008 July and 24 mid-eclipse times were measured (see those listed Table 1). The $O-C$ values were determined by the observed $(O)$ mid-eclipse times minus the calculated $(C)$ times with the ephemeris published by Baptista et al. (2003),

$$
\text { Min. I = BJD } 2443245.97694+0.0624278634 \times E,
$$

and $E$ is the cycle number. The corresponding $O-C$ diagram is shown in Figure 5. A parabolic change of the $O-C$ curve corresponds to a linear period change.

We found that the newly determined mid-eclipse times do not follow the previous general trend of the cyclic change, indicating that a simple cyclic variation cannot fit the $O-C$ curve satisfactorily (see Figure 5). To describe the general trend of the $O-C$ diagram well, a downward parabolic change (dashed line in Figure 5) is required apart from the smallamplitude cyclic variation reported previously (Baptista et al. 2003). The downward parabolic change reveals a longterm period decrease. Since the cyclic oscillation of the $O-C$ diagram may be caused by light travel time effect via the presence of a third body (e.g., Marsh et al. 2014), we
Table 2

Orbital Parameters of the Giant Circumbinary Planet

\begin{tabular}{lc}
\hline \hline Parameters & Values \\
\hline Revised epoch, $\Delta T_{0}$ (days) & $-0.000177( \pm 0.000050)$ \\
Revised period, $\Delta P_{0}$ (days) & $+1.68( \pm 0.73) \times 10^{-9}$ \\
Rate of the period change, $P$ (days $\left.\mathrm{yr}^{-1}\right)$ & $-5.93( \pm 0.81) \times 10^{-9}$ \\
Eccentricity, $e$ & $0.37( \pm 0.21)$ \\
Longitude of the periastron passage, $\omega(\mathrm{deg})$ & $190.2( \pm 16.9)$ \\
Periastron passage, $T$ & $2452955.9( \pm 445.4)$ \\
The semi-amplitude, $K$ (days) & $0.000329( \pm 0.000059)$ \\
Orbital period, $P_{3}($ years $)$ & $21.64( \pm 0.67)$ \\
Projected semimajor axis, $a_{12} \sin i^{\prime}(\mathrm{AU})$ & $0.057( \pm 0.010)$ \\
Mass function, $f(m)\left(M_{\odot}\right)$ & $3.96( \pm 0.71) \times 10^{-7}$ \\
Mass of the third companion, $M_{3} \sin i^{\prime}\left(M_{\odot}\right)$ & $0.00703( \pm 0.00071)$ \\
Orbital separation, $d_{3}\left(i^{\prime}=90^{\circ}\right)(\mathrm{AU})$ & $9.0( \pm 1.0)$ \\
\hline
\end{tabular}

considered a general case with an eccentric orbit (e.g., Irwin 1952). The following equation,

$$
O-C=\Delta T_{0}+\Delta P_{0} \times E+\beta E^{2}+\tau,
$$

was used to describe the $O-C$ diagram. $\Delta T_{0}$ and $\Delta P_{0}$ in the equation are the revised epoch and orbital period. $\beta$ is the rate of the linear period decrease and $\tau$ is the cyclic variation caused by the presence of the third body, i.e.,

$$
\begin{aligned}
\tau & =K\left[\left(1-e^{2}\right) \frac{\sin (\nu+\omega)}{1+e \cos \nu}+e \sin \omega\right] \\
& =K\left[\sqrt{1-e^{2}} \sin E^{*} \cos \omega+\cos E^{*} \sin \omega\right] .
\end{aligned}
$$

$\nu$ and $E^{*}$ are the true anomaly and the eccentric anomaly, respectively. $K=\frac{a_{12} \sin i^{\prime}}{c}\left(a_{12} \sin i^{\prime}\right.$ is the projected semimajor axis). While solving Equation (3), the two following equations were applied:

$$
N=E^{*}-e \sin E^{*}
$$

and

$$
N=\frac{2 \pi}{P_{3}}(t-T)
$$

$N$ is the mean anomaly while $t$ is the time of light minimum. The other parameters and the corresponding values are described in Table 2.

The result indicates that the orbital period of V2051 Oph shows a cyclic change as it undergoes a long-term decrease. The rate of the long-term decrease is $\dot{P}=-5.93 \times 10^{-10}$ days $\mathrm{yr}^{-1}=-1.62 \times 10^{-12} \mathrm{~s} \mathrm{~s}^{-1}$ (or $1 \mathrm{~s}$ in about 19,600 years). The amplitude and the period of the cyclic oscillation are about $28.4 \mathrm{~s}$ and 21.64 years, respectively. The derived parameters of the cyclic change are close to those determined by Baptista et al. (2003). After both of the variations are subtracted, the residuals are plotted in the lower panel of Figure 5.

\section{DISCUSSIONS}

V2051 Oph is a short-period dwarf nova below the CV period gap. According to the standard model, the evolution of CVs below the period gap is driven by GR (Rappaport et al. 1983; Spruit \& Ritter 1983). The contribution of GR to the period decrease was given by Kraft et al. (1962) and 
Faulkner (1971) as

$$
\frac{\dot{P}_{1}}{P}=-3 \frac{32 G^{3}}{5 c^{5}} \frac{M_{1} M_{2}\left(M_{1}+M_{2}\right)}{d^{4}} .
$$

$P$ is the orbital period while $M_{1}$ and $M_{2}$ are the masses of the primary and secondary components, respectively. $d$ is the orbital separation, $G$ and $c$ are the gravitational constant and the speed of light. For V2051 Oph, a computation with the equation yields $\dot{P}_{1}=-1.00 \times 10^{-10}$ days $\mathrm{yr}^{-1}$ using the parameters given by Baptista et al. (1998), i.e., $M_{1}=0.78 M_{\odot}$, $M_{2}=0.15 M_{\odot}$, and $d=0.64 R_{\odot}$. This is a few times less than the observed rate in the orbital period $(\dot{P}=$ $-5.93 \times 10^{-10}$ days $\mathrm{yr}^{-1}$ ), indicating that GB is insufficient to explain the observed period change. Additional angular momentum losses are required to produce the rate of period decrease.

In the standard theory, to explain the $\mathrm{CV}$ period gap, $\mathrm{MB}$ was assumed to stop at an orbital period close to $3 \mathrm{hr}$ when the secondary component becomes completely convective (Rappaport et al. 1983; Spruit \& Ritter 1983). However, recent investigations suggested that fully convective stars are very active (e.g., Delfosse et al. 1998). They are also strongly and stably magnetic (e.g., Saar \& Linsky 1985; Johns-Krull \& Valenti 1996; Reiners \& Basri 2007; Morin et al. 2008). The assumption of disrupted magnetic braking in the standard theory of CVs is in conflict with the activity data. Moreover, the AML rate deduced from the rotation rates observed in young open clusters shows that the braking rate is continuous over the fully convective boundary (Andronov et al. 2003). The long-term decrease in the orbital period of V2051 Oph supports the conclusion that fully convective stars may be also undergoing secular AML through MB.

The period decrease rate caused by additional AML via MB is $\dot{P}_{2}=\dot{P}-\dot{P}_{1}=-4.93 \times 10^{-10}$ days $\mathrm{yr}^{-1}$. It is shown that the magnetic braking rate is about 4.9 times the gravitational radiation rate. Using the equation (Tout \& Hall 1991)

$$
\frac{\dot{P_{2}}}{P}=-2\left(\frac{R_{\mathrm{A}}}{d}\right)^{2} \frac{M}{M_{1} M_{2}} \dot{M}
$$

a calculation leads to $R_{\mathrm{A}}{ }^{2} \dot{M}=2.03 \times 10^{-10}$ in units of $R_{\odot}{ }^{2} M_{\odot} \mathrm{yr}^{-1} \cdot R_{\mathrm{A}}$ in the equation is the Alfvén radius. The relation between the mass loss rate $(\dot{M})$ and the Alfvén radius $\left(R_{\mathrm{A}}\right)$ is shown in Figure 6. If $R_{\mathrm{A}}=15 R_{2}=2.4 R_{\odot}$, then the mass loss rate is $\dot{M}=3.5 \times 10^{-11} M_{\odot} \mathrm{yr}^{-1}$. When the Alfvén radius equals that of the $\operatorname{Sun}$ (i.e., $R_{\mathrm{A}}=15 R_{\odot}$ ), then the required mass loss rate is $\dot{M}=9.02 \times 10^{-13} M_{\odot}$ per year. These values are smaller than the typical rate of mass transfer driven by gravitational radiation $\left(M_{\text {tran }} \sim 4 \times 10^{-11} M_{\odot} \mathrm{yr}^{-1}\right.$; e.g., Schaefer et al. 2010).

The cyclic period oscillations of close binaries comprised of one or two cool stars were explained by magnetic activity cycles (Applegate 1992). This mechanism was proposed based on the conclusion reported by Hall (1989), who analyzed the orbital period changes of 101 Algols. The author found that all cases of cyclic period changes are restricted to binary systems with cool secondary components (i.e., spectral types later than F5). However, a recent statistical investigation of the cyclic period changes has shown that the conclusion proposed by Hall is not correct. The percentages of cyclic changes are similar for both late-type and early-type close binary stars, indicating that

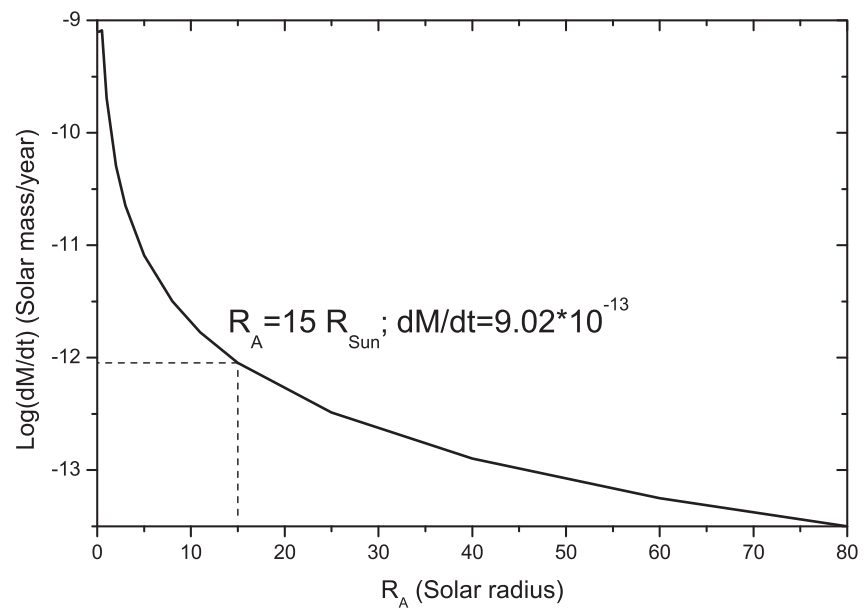

Figure 6. Relation between the mass loss rate and the Alfvén radius in order to cause the continuous decrease in the orbital period due to MB. After subtracting the contribution of gravitational radiation, the contribution of magnetic braking to the period decrease is $\dot{P}_{2}=-4.93 \times 10^{-10} \mathrm{days} \mathrm{yr}^{-1}$ (or $1 \mathrm{~s}$ in about 23,500 years). Therefore, we derive $R_{\mathrm{A}}{ }^{2} \dot{M}=2.03 \times 10^{-10}$ in units of $R_{\odot}{ }^{2} M_{\odot} \mathrm{yr}^{-1}$. When the Alfvén radius equals that of the Sun $\left(R_{A}=15 R_{\odot}\right)$, the derived mass loss rate in the stellar wind is $\dot{M}=9.02 \times 10^{-13} M_{\odot}$ per year.

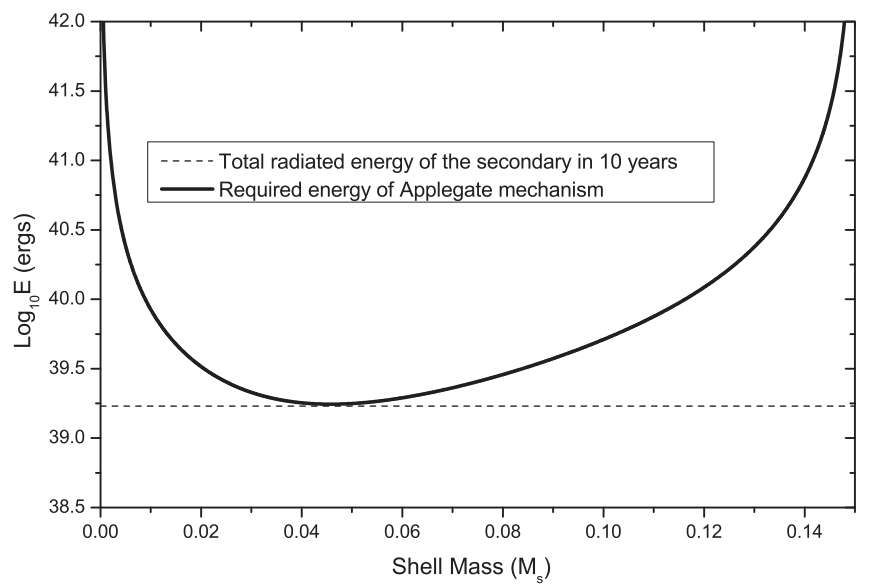

Figure 7. Energy required to produce the cyclic oscillation in the $O-C$ diagrams using Applegate's mechanism (solid line). $M_{\mathrm{s}}$ is the assumed shell mass of the cool component. The dashed line represents the total energy that radiates from the secondary in 10 years.

the plausible explanation of the cyclic period variations is the light travel-times effect via the presence of a third body (Liao \& Qian 2010).

According to the Applegate mechanism, some angular momentum is periodically transferred between different parts in the convection zone of the cool component star. Therefore the rotational oblateness will change, which causes the orbital period to be variable while the cool star goes through its magnetic activity cycles. As for V2051 Oph, the secondary is a fully convective and rapidly rotating star. It has no thin transition between the convective envelope and radiative core where dynamo processes are produced for solar-type stars (Barnes et al. 2005). However, it presumably hosts dynamo processes (Chabrier \& Küker 2006). Using the same method as in Brinkworth et al. (2006), the energies required to cause the cyclic period change were computed and are displayed in Figure 7. We show that the required energies are larger than the total energy radiated from the fully convective star in 10 years. 
This suggests that the Applegate mechanism cannot explain the cyclic variation. A temperature of $T_{2}=2800 \mathrm{~K}$ for the secondary was used during the calculation and the luminosity was computed using $L_{2}=\left(\frac{R_{2}}{R_{\odot}}\right)^{2}\left(\frac{T_{2}}{T_{\odot}}\right)^{4} L_{\odot}$.

Therefore, the cyclic period oscillation can plausibly be explained by the wobble of the binary's barycenter via the existence of a third body. With the absolute parameters $M_{1}=0.78 M_{\odot}$ and $M_{2}=0.15 M_{\odot}$ (Baptista et al. 1998), a computation using the equation

$$
f(m)=\frac{4 \pi^{2}}{G P_{3}^{2}} \times\left(a_{12}^{\prime} \sin i^{\prime}\right)^{3}=\frac{\left(M_{3} \sin i^{\prime}\right)^{3}}{\left(M_{1}+M_{2}+M_{3}\right)^{2}},
$$

leads to the mass function and the mass of the tertiary companion as $f(m)=3.96( \pm 0.71) \times 10^{-7} M_{\odot}$ and $M_{3} \sin i^{\prime}=0.0070( \pm 0.0007) M_{\odot}=7.3( \pm 0.7) M_{\text {Jup }}$, respectively. $G$ in the equation is the gravitational constant, while $P_{3}$ is the period of the $(O-\mathrm{C})_{2}$ oscillation. The projected radius of V2051 Oph orbiting the barycenter of the triple system can be obtained with the following equation:

$$
a_{12}^{\prime} \sin i^{\prime}=K \times c .
$$

$K$ is the amplitude of the cyclic change and $c$ is the speed of light. When $i^{\prime} \geqslant 30^{\circ} .3$, its mass corresponds to $0.0070 M_{\odot} \leqslant M_{3} \leqslant 0.0140 M_{\odot}$, and it should be a giant extrasolar planet (Chabrier \& Baraffe 2000; Burrows et al. 2001). However, depending on the unknown inclination, it could be a brown dwarf and a low-mass star (i.e., $5.86 \leqslant i^{\prime} \leqslant 30^{\circ} .3$ for a brown dwarf or $i^{\prime} \leqslant 5^{\circ} .86$ for a lowmass star).

\section{CONCLUSIONS}

By photometrically monitoring V2051 Oph for about seven years, we discovered that, in addition to a cyclic change, the orbital period shows a long-term decrease. Its rate is $\dot{P}=-5.93 \times 10^{-10}$ days $\mathrm{yr}^{-1}$. This rate is about five times larger than that caused by gravitational radiation. To solve the problem of the period minimum of CVs encountered in the standard CV theory, additional AML is required for the systems below the period gap, which is about several times the gravitational radiation rate (Kolb \& Baraffe 1999; Patterson 2001; Kolb 2002). Moreover, based on the investigation of a homogeneous $\mathrm{CV}$ sample (selected for $\mathrm{H}_{\alpha}$ emission), it was deduced that $\mathrm{CVs}$ below the period gap should evolve faster than predicted by the standard theory, implying that another mechanism for AML besides gravitational radiation is present (Pretorius \& Knigge 2008). Our study suggests that the mechanism causing the additional AML may be magnetic stellar wind braking of the fully convective star. A detailed calculation has shown that the additional AML rate should be up to five times the gravitational radiation rate (King et al. 2002), which is in good agreement with our result.

We conclude that the observed period decrease in V2051 Oph is caused by a combination of the GR and MB of the fully convective component star. This conclusion reveals that magnetic braking may not completely stop for fully convective stars. Few CVs were found with periods below about 78 minutes, which may actually be caused by the cessation of magnetic braking when the secondary becomes a brown dwarf that has an extremely weak magnetic field compared with active M-type dwarfs (e.g., Berger et al. 2001). To check our conclusion, continuous monitoring of other deeply eclipsing dwarf novae below the period gap is required. If magnetic braking is not completely stopped for fully convective stars, then this kind of fast orbital decay should be observed for other ultrashort-period dwarf novae.

Since the Applegate mechanism has difficulty explaining the cyclic change in the $O-C$ diagram, it was interpreted as being due to the existence of a third body. As displayed in the upper panel of Figure 5, the data cover nearly two cycles of the periodic change that supports this explanation. It is discovered that the third body in V2051 Oph is a circumbinary planet with $66.3 \%$ probability by assuming a random distribution of orbital inclination. Investigations of exoplanets in the last 23 years have shown that they can exist almost anywhere. Their host stars could be of all kinds. The discovery of a giant planet orbiting the dwarf nova V2051 Oph indicates that dwarf novae are also a kind of star hosting planets. This will help us to understand the formation and evolution of planets. Some progenitors of CVs, such as the sdB-type star NY Vir (e.g., Qian et al. 2012b; Lee et al. 2014) and the detached white dwarf binary NN Ser (e.g., Marsh et al. 2014), were determined to be hosting exoplanets. After they evolve into CVs through AML via GR and $M B$, they still keep their circumbinary planets. We expect that both CVs and their progenitors host substellar objects and that those substellar objects could be used as a "tracer" for CV formation and evolution. Morever, as in the case of NY Vir (Qian et al. 2012b), the observed downward parabolic change in the $O-C$ diagram (the dashed line in the upper panel of Figure 5) may be only a part of a long-period (longer than 38 years) cyclic change. This cyclic variation may be caused by the light travel time effect due to the presence of another circumbinary planet $\left(M_{4} \sim 5 M_{\text {Jup }}\right)$ in a wider orbit.

This work is partly supported by Chinese Natural Science Foundation (No.11133007 and No. 11325315), the Key Research Program of the Chinese Academy of Sciences (grant No. KGZD-EW-603), the Science Foundation of Yunnan Province (No. 2012HC011 and 2013FB084), and by the Strategic Priority Research Program "The Emergence of Cosmological Structures" of the Chinese Academy of Sciences (No. XDB09010202). New CCD photometric observations of V2051 Oph were obtained with the $2.4-\mathrm{m}$ and the $1.0-\mathrm{m}$ telescopes in Yunnan Observatories and the 0.6-m Helen Sawyer Hogg telescope and the 2.15-m Jorge Sahade telescope in Argentina.

\section{REFERENCES}

Almeida, L. A., \& Jablonski, F. 2011, IAUS, 276, 495

Andronov, N., Pinsonneault, M., \& Sills, A. 2003, ApJ, 582, 358

Applegate, J. H. 1992, ApJ, 385, 621

Backhaus, U., Bauer, S., Beuermann, K., et al. 2012, A\&A, 538, A84

Baptista, R., Borges, B. W., Bond, H. E., et al. 2003, MNRAS, 345, 889

Baptista, R., Catalan, M. S., Horne, K., \& Zilli, D. 1998, MNRAS, 300, 233

Baptista, R., Santos, R. F., Fandez-Abans, M., \& Bortoletto, A. 2007, AJ, 134,867

Barnes, J. R., Cameron, A. C., Donati, J. F., et al. 2005, MNRAS, 357, L1

Berger, E., Ball, S., Becker, K. M., et al. 2001, Natur, 410, 338

Beuermann, K., Buhlmann, J., Diese, J., et al. 2011, A\&A, 526, A53

Brinkworth, C. S., Marsh, T. P., Dhillon, V. S., \& Knigge, C. 2006, MNRAS, 365, 287

Burleigh, M. R., Clarke, F. J., Hogan, E., et al. 2008, MNRAS, 386, L5 
Burrows, A., Hubbard, W. B., Lunine, J. T., \& Liebert, J. 2001, RvMP, 73,719

Carlberg, J. K., Majewski, S. R., \& Arras, P. 2009, ApJ, 700, 832

Chabrier, G., \& Baraffe, I. 2000, ARA\&A, 38, 337

Chabrier, G., \& Küker, M. 2006, A\&A, 446, 1027

Delfosse, X., Forveille, T., Perrier, C., \& Mayor, M. 1998, A\&A, 331, 581

Farihi, J., Becklin, E. E., \& Zuckerman, B. 2008, ApJ, 681, 1470

Faulkner, J. 1971, ApJ, 170, 99

Goździewski, K., Slowikowska, A., Dimitrov, D., et al. 2015, MNRAS, 448, 1118

Hall, D. S. 1989, SSRv, 50, 219

Hogan, E., Burleigh, M. R., \& Clarke, F. J. 2009, MNRAS, 396, 2074

Howard, A. W., Marcy, G. W., Johnson, J. A., et al. 2010, Sci, 330, 653

Irwin, J. B. 1952, ApJ, 116, 211

Johns-Krull, C. M., \& Valenti, J. A. 1996, ApJL, 459, L95

Kilic, M., Stanek, K. Z., \& Pinsonneault, M. H. 2007, ApJ, 671, 761

King, A. R., Schenker, K., \& Hameury, J. M. B. 2002, MNRAS, 335, 513

Kolb, U. 1993, A\&A, 271, 149

Kolb, U. 2002, in ASP Conf. Ser. 261, The Physics of Cataclysmic Variables and Related Objects, ed. B. T. Gänsicke et al. (San Francisco, CA: ASP), 180

Kolb, U., \& Baraffe, I. 1999, MNRAS, 309, 1034

Kraft, R. P., Matthews, J., \& Greenstein, J. L. 1962, ApJ, 136, 312

Lee, J. W., Hinse, T. C., Youn, J.-H., \& Han, W. 2014, MNRAS, 445, 2331

Liao, W.-P., \& Qian, S.-B. 2010, MNRAS, 405, 1930

Marsh, T. R., Parsons, S. G., Bours, M. C. P., et al. 2014, MNRAS, 437, 475

Morin, J., Donati, J. F., Forveille, T., et al. 2008, MNRAS, 384, 77
Mullally, F., Kilic, M., Reach, W. T., et al. 2007, ApJS, 171, 206

Mustill, A. J., \& Villaver, E. 2012, ApJ, 761, 121

Nordhaus, J., Spiegel, D. S., Ibgui, L., Goodman, J., \& Burrows, A. 2010, MNRAS, 408, 631

Patterson, J. 2001, PASP, 113, 736

Pretorius, M. L., \& Knigge, C. 2008, MNRAS, 38, 1485

Pribulla, T., Vaňko, M., Ammler-von Eiff, M., et al. 2012, AN, 333, 754

Qian, S.-B., Dai, Z.-B., Liao, W.-P., et al. 2009a, ApJL, 706, L96

Qian, S.-B., Liao, W.-P., Zhu, L.-Y., \& Dai, Z.-B. 2010a, ApJL, 708, L66

Qian, S.-B., Liao, W.-P., Zhu, L.-Y., et al. 2010b, MNRAS, 401, L34

Qian, S.-B., Liu, L., Liao, W.-P., et al. 2011, MNRAS, 414, L16

Qian, S.-B., Liu, L., Zhu, L.-Y., et al. 2012a, MNRAS, 422, L24

Qian, S.-B., Shi, G., Zola, S., et al. 2013, MNRAS, 436, 1408

Qian, S. B., Soonthornthum, B., Dai, Z. B., et al. 2009b, ASPC, 404, 248

Qian, S.-B., Zhu, L.-Y., Dai, Z.-B., et al. 2012b, ApJL, 745, L23

Qian, S.-B., Zhu, L.-Y., Zola, S., et al. 2009c, ApJL, 695, L163

Rappaport, S., Verbunt, F., \& Joss, P. C. 1983, ApJ, 275, 713

Reiners, A., \& Basri, G. 2007, ApJ, 656, 1121

Saar, S. H., \& Linsky, J. L. 1985, ApJL, 299, L47

Saito, R. K., \& Baptista, R. 2006, AJ, 131, 2185

Sanduleak, N. 1972, IBVS, 663

Schaefer, B. E., Pagnotta, A., \& Shara, M. M. 2010, ApJ, 708, 381

Spruit, H. C., \& Ritter, H. 1983, A\&A, 124, 267

Tout, C. A., \& Hall, D. S. 1991, MNRAS, 253, 9

Villaver, E., \& Livio, M. 2007, ApJ, 661, 1192

Warner, B. 1995, Cataclysmic Variable Stars Cambridge Astrophysics Series (Cambridge: Cambridge Univ. Press), 28

Wood, J. H., Irwin, M. J., \& Pringle, J. E. 1985, MNRAS, 214, 475 